\title{
A retrospective computed tomography analysis of maxillary fractures and the clinical outcomes of their unreduced parts
}

\author{
Chan Min Chung ${ }^{1}$, \\ Seung Wan Tak ${ }^{1}$, \\ Hyoseob Lim ${ }^{1}$, \\ Sang Hun Cho ${ }^{1}$, \\ Jong Wook Lee ${ }^{2}$ \\ ${ }^{1}$ Department of Plastic and \\ Reconstructive Surgery, Hallym \\ University Sacred Heart Hospital, \\ Hallym University College of Medicine, \\ Anyang; ${ }^{2}$ Department of Plastic and \\ Reconstructive Surgery, Hallym \\ University Hangang Sacred Heart \\ Hospital, Hallym University College of \\ Medicine, Seoul, Korea
}

\begin{abstract}
Background: Some parts of a maxillary fracture - for example, the medial and posterior wallsmay remain unreduced because they are unapproachable or hard to deal with. This study aimed to investigate the self-healing process of unreduced maxillary membranous parts of fractures through a longitudinal computed tomography (CT) analysis of cases of unilateral facial bone injuries involving the maxillary sinus walls.

Methods: Thirty-two patients who had undergone unilateral facial bone reduction surgery involving the maxillary sinus walls without reduction of the medial and posterior walls were analyzed in this retrospective chart review. Preoperative, immediate postoperative, and 3-month postoperative CT scans were analyzed. The maxillary sinus volume was calculated and improvements in bone continuity and alignment were evaluated.

Results: The volume of the traumatized maxillary sinuses increased after surgery, and expanded significantly by 3 months postoperatively $(p<0.05)$. The significant preoperative volume difference between the normal and traumatized sides $(p=0.024)$ resolved after surgery $(p>0.05)$, and this resolution was maintained at 3 months postoperatively $(p>0.05)$. The unreduced parts of the maxillary bone showed improved alignment and continuity (in $75.0 \%$ and $90.6 \%$ of cases, respectively), and improvements in bone alignment and bone continuity were found to be correlated using the Pearson chi-square test ( $p=0.002)$.

Conclusion: Maxillary wall remodeling through self-healing occurred concomitantly with an increase in sinus volume and simultaneous improvements in bone alignment and continuity. Midfacial surgeons should be aware of the natural course of unreduced fractured medial and posterior maxillary walls in complex maxillary fractures.
\end{abstract}

Keywords: Facial bones / Fractures, bone / Fracture healing / Maxillary sinus / Periosteum

\section{INTRODUCTION}

The paranasal sinuses have various functions. For instance,

\section{Correspondence: Hyoseob Lim}

Department of Plastic and Reconstructive Surgery, Hallym University Sacred

Heart Hospital, Hallym University College of Medicine, 22 Gwanpyeong-ro

170beon-gil, Dongan-gu, Anyang 14068, Korea

E-mail: hyoseob.lim@gmail.com

Received September 25, 2019 / Revised December 9, 2019/

Accepted December 13, 2019 they reduce the weight of the skull by constituting areas of empty space in the skull. Additionally, active or passive ventilation of the paranasal sinuses can cool the brain [1], and specific pairs of the paranasal sinuses can control voice resonance, modulate intranasal gas pressure, and drain secretions from the mucosa of the nasal cavity through the mucociliary effect $[2,3]$. Furthermore, the paranasal sinuses serve as buffers against facial trauma, as they help distribute the force of external impacts. These sinuses also protect dental roots and the nasal cavity. 
However, because of the thin bony structures of maxillary sinuses - unlike the bones of the extremities - maxillary sinus fractures can easily occur due to external trauma [4]. Facial trauma is most commonly caused by violence, slipping and falling, traffic accidents, and sports activities [5].

Of the paranasal sinuses, the maxillary sinus is most commonly treated by oral and maxillofacial surgeons. Thus, having a solid understanding of the anatomical features and characteristics of this region is of fundamental importance. The maxillary sinus frequently has multiple cavities that are divided by several septa [6]. Rancitelli et al. [7] reported that $38.1 \%$ of the maxillary sinuses they investigated contained septa. It is also important to consider the distribution of the arterial supply to minimize postoperative complications [8-10]. Preserving special structures such as septa or the Schneiderian membrane and avoiding further injury of the arteries supplying the maxillary sinus are major concerns for surgeons.

Reduction techniques are commonly used to rebuild the original location and shape of the facial bone. However, some facial structures are difficult to approach, meaning that some parts of the fractures remain unreduced; for example, the medial and posterior walls of the maxillary sinus often cannot be reduced because of their location. It is widely accepted that only maxillary buttress reconstruction is sufficient for midface surgery. However, the natural course of unreduced fractured maxillary thin walls has not been clearly characterized. For this reason, we conducted this study to observe maxillary thin wall remodeling during the postoperative period.

The aim of this study was to investigate the self-healing process of unreduced midfacial bones through a longitudinal analysis of computed tomography (CT) scans of patients who underwent unilateral facial bone injuries involving the maxillary sinus walls.

\section{METHODS}

\section{Study subjects}

The subjects of this investigation were selected by chart review among patients who had undergone unilateral facial bone reduction surgery involving the maxillary sinus walls. All the patients had undergone surgery under general anesthesia by open reduction and internal fixation with titanium or absorbable plates and screws, without reduction of the medial and posterior walls of the maxillary sinus. The inclusion criteria were as follows: (1) no prior fracture history of the facial bones; (2) no old fracture identified on facial CT; (3) no history of maxillary sinusitis, wound infection, or further trauma during the 3-month postoperative period, as those conditions might affect the natural healing process after surgery; and (4) the availability of preoperative, immediate postoperative, and 3-month postoperative facial CT scans in their chart. To be more specific regarding the last inclusion criterion, not all of the patients received long-term postoperative facial CT follow-up. Long-term postoperative CT follow-up was only performed for patients who wanted their facial bone structures to be checked on CT. Scans were not obtained before 3 months postoperatively because bone healing might have still been taking place. The period between surgery and the long-term follow-up CT scans was between 3 months and 8 months, and 3-month postoperative CT scans accounted for the largest proportion of cases in the retrospective chart review. The exclusion criterion was bilateral midfacial fracture. The severity of the fracture, sex, age, mode of trauma, specific surgical technique, and involvement of other facial bone fractures were not considered. We had no exclusion criteria regarding the subtypes of zygomaticomaxillary complex fractures; therefore, all fracture types were included in the study.

\section{CT analysis}

Maxillary sinus volume and improvements in bone alignment and continuity were compared on preoperative, immediate postoperative (within 2 weeks), and 3-month follow-up CT scans. To investigate the self-healing process of the unreduced maxillary sinus walls, CT volumetry was performed on both the injured and uninjured sides. For the injured side, preoperative, immediate postoperative, and 3-month postoperative volumetry was performed using the INFINITT PACS M6 software (INFINITT Healthcare Co., Ltd., Seoul, Korea). We calculated the maxillary sinus area two-dimensionally, and multiplied it by the thickness between each cut ( $3 \mathrm{~mm}$ ) (Fig. 1). Technically, in-

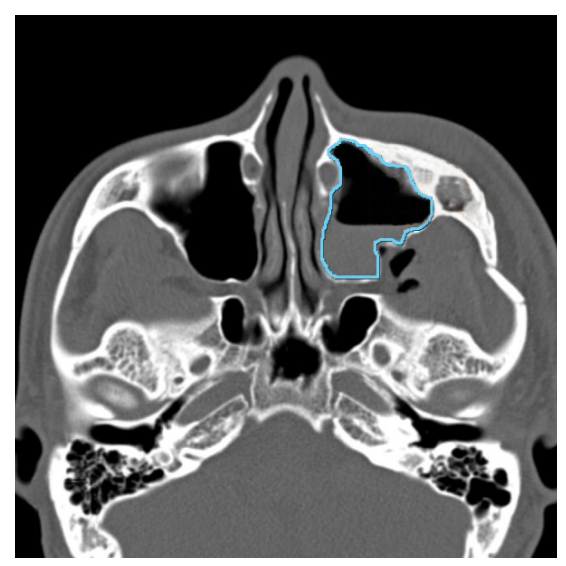

Fig. 1. Volumetric measurements made using the INFINITT PACS M6. The two-dimensional area of the axillary sinus was calculated in each image cut, and multiplied by $3 \mathrm{~mm}$ (the thickness between image cuts) to obtain the maxillary sinus volume (blue line). 
tegral calculus was used to estimate maxillary sinus volume. The volume of both the injured and uninjured sides was calculated at the preoperative, immediate postoperative, and 3 -month postoperative time points. Next, improvements in the bone alignment and bone continuity of the unreduced medial and posterior walls of the maxillary sinus were investigated by two plastic surgeons (HL and SWT) (Fig. 2). Bone alignment improvement was defined as a decrease in the extent of bony fragments, with a tendency for them to regain their original shape and linear contour. Bone continuity improvement was defined as the inability of the investigators to find the fracture line due to bone healing. Bone alignment and bone continuity improvement were graded as either improved or not improved. Improvement was only considered to have taken place if both investigators concurred.

\section{Statistical analysis}

Data were analyzed using SPSS for Windows version 24.0 (IBM Corp., Armonk, NY, USA). The level of statistical significance was set at $p<0.05$. The volume of the normal side was measured, and the volume of the traumatized side was measured at the preoperative, immediate postoperative, and 3-month postoperative time points. The pairwise difference between each

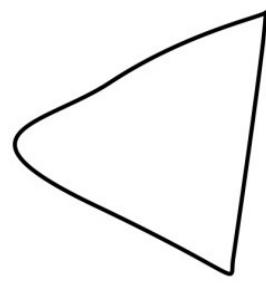

Schematic drawing of maxillary sinus

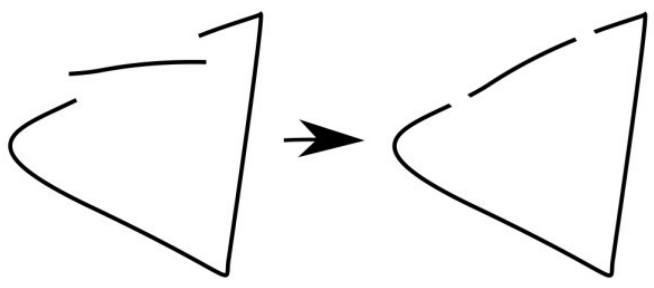

Improvement of bone alignment

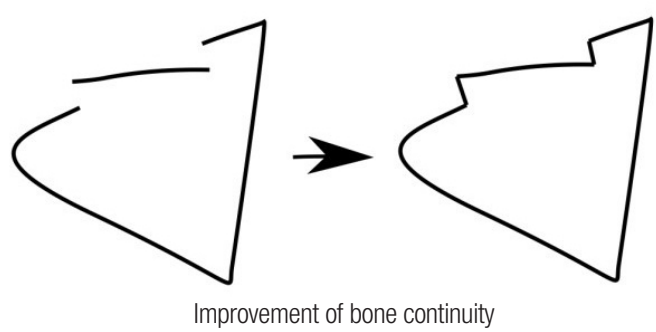

Fig. 2. Schematic drawings illustrating the definition of improvements in bone continuity and bone alignment. category of volume measurements was analyzed using the paired $t$-test. The relationship between improvements in bone alignment and bone continuity was analyzed using the Pearson chi-square test.

\section{Ethical approval}

The study was approved by the Institutional Review Board of Hallym University Sacred Heart Hospital (IRB No. 2018-05035-001) and performed in accordance with the principles of the Declaration of Helsinki.

\section{RESULTS}

Thirty-two patients (five men and 27 women) with preoperative, immediate postoperative, and 3-month postoperative facial CT data in their charts were included in this study. Their demographic features are shown in Table 1. Each category of volume measurements was defined as follows: $\mathrm{NL}$, normal side volume; TS, traumatized side volume (preoperative); TSI, traumatized side volume (immediate postoperative); TS3, traumatized side volume ( 3 months postoperatively). The average maxillary sinus volume values were as follows: $\mathrm{NL}, 24.98 \pm 8.64$ $\mathrm{mL}$; TS, $23.10 \pm 8.00 \mathrm{~mL}$; TSI, $25.09 \pm 7.88 \mathrm{~mL}$; TS3, $25.49 \pm 8.06$ $\mathrm{mL}$ (Table 2).

All possible pairs of the four categories of volume measurements were compared using the paired $t$-test (Table 3$)$. TS was significantly smaller than NL by $1.88 \pm 4.47 \mathrm{~mL}(p=0.024)$, while the other two postoperative values (TSI and TS3) showed no significant difference compared to NL. Of the traumatized

Table 1. Demographic features of all patients with unilateral maxillary fractures

\begin{tabular}{lc}
\hline Variable & Value $(\mathrm{n}=32)$ \\
\hline Sex & \\
Male & $5(15.62)$ \\
Female & $27(84.38)$ \\
Age $(\mathrm{yr})$ & $37.25 \pm 18.34$ \\
$10-29$ & $14(43.75)$ \\
$30-49$ & $11(34.38)$ \\
$50-69$ & $6(18.75)$ \\
$70-89$ & $1(3.13)$ \\
\hline
\end{tabular}

Values are presented as number (\%) or mean \pm standard deviation.

Table 2. Volumetric data of computed tomography scans

\begin{tabular}{ccccc}
\hline & NL & TS & TSI & TS3 \\
\hline Mean \pm SD (mL) & $24.98 \pm 8.64$ & $23.10 \pm 8.00$ & $25.09 \pm 7.88$ & $25.49 \pm 8.06$ \\
\hline
\end{tabular}

NL, normal side volume; TS, traumatized side volume (preoperative); TSI, traumatized side volume (immediate postoperative); TS3, traumatized side volume (3 months postoperatively); SD, standard deviation. 
side values, TSI was larger than TS by $1.99 \pm 2.35 \mathrm{~mL}$, TS3 was larger than TS by $2.39 \pm 2.23 \mathrm{~mL}$, and TS3 was larger than TSI by $0.41 \pm 1.12 \mathrm{~mL}$, all with statistical significance $(p=0.000$, $p=0.000, p=0.048$, respectively).

Twenty-four patients (75\%) showed improved bone alignment 3 months after surgery, and 29 patients (90.6\%) showed improved continuity during the same period (Fig. 3). A significant correlation was found between improvements in bone alignment and bone continuity by the Pearson chi-square test $(p=0.002)($ Table 4$)$.

\section{DISCUSSION}

Maxillary thin wall remodeling was observed during the postoperative period. The volume of the traumatized maxillary sinuses expanded gradually after surgery, with statistical significance $(p<0.05)$. Furthermore, the significant preoperative volume difference between the normal side and traumatized side (TS-NL, $p=0.024$ ) resolved after surgery (TSI-NL, $p>0.05$ ), and this recovery was maintained at 3 months postoperatively

Table 3. Comparisons between computed tomography volumetry values

\begin{tabular}{lccl}
\hline Variable & Mean $\pm \mathrm{SD}(\mathrm{mL})$ & $95 \% \mathrm{Cl}$ & $p$-value \\
\hline TS-NL & $1.88 \pm 4.47$ & -3.49 to -0.27 & $0.024^{\mathrm{b})}$ \\
TSI-NL & $0.11 \pm 3.10$ & -1.01 to 1.23 & 0.843 \\
TS3-NL & $0.52 \pm 3.48$ & -0.74 to 1.77 & 0.408 \\
TSI-TS & $1.99 \pm 2.35$ & 1.14 to 2.84 & $0.000^{\text {b) }}$ \\
TS3-TS & $2.39 \pm 2.23$ & 1.59 to 3.20 & $0.000^{\text {b) }}$ \\
TS3-TSI & $0.41 \pm 1.12$ & 0.00 to 0.80 & $0.048^{\text {b) }}$ \\
\hline
\end{tabular}

SD, standard deviation; $\mathrm{Cl}$, confidence interval; TS, traumatized side volume (preoperative); NL, normal side volume; TSI, traumatized side volume (immediate postoperative); TS3, traumatized side volume (3 months postoperatively).

a)Paired $t$-test; ${ }^{b}$ Statistically significant, $p<0.05$.
(TS3-NL, $p>0.05$ ). Furthermore, the unreduced parts of the maxillary bone showed improved alignment and continuity (in $75.0 \%$ and $90.6 \%$ of cases, respectively), meaning that the maxillary thin wall contour improved. In the statistical analysis, bone alignment and bone continuity were correlated with each other, implying that these two processes occurred simultaneously. Maxillary wall remodeling took place through self-healing in the patients analyzed in this study, with larger sinus volume and simultaneous improvements in bone alignment and continuity.

In the present study, the posterior lateral wall and medial wall of the maxillary sinus showed improvements despite not being surgically approachable. Additionally, the anterior wall, which is surgically approachable, showed a better contour at 3 months after surgery. One possible explanations for this self-healing process relates to the fact that facial bones heal by direct ossification of the mesenchyme, unlike the other bones of the skeleton, which healed via preformed cartilage through the process of endochondral ossification [11]. Osseous defect healing, bone regeneration, and the implant-bone interface have previously been investigated in a few articles [12-14]. Additionally, other factors can affect facial bone healing. Esteve-Altava and Rasskin-Gutman [15] claimed that bone in the craniofacial area is regulated

Table 4. Relationship between bone alignment and bone continuity improvement $^{\text {a) }}$

\begin{tabular}{llccc}
\hline & & \multicolumn{3}{c}{ Continuity } \\
\cline { 3 - 5 } & & Not improved & Improved & Total \\
\hline \multirow{2}{*}{ Alignment } & Not improved & 3 & 5 & 8 \\
& Improved & 0 & 24 & 24 \\
& Total & 3 & 29 & 32 \\
\hline
\end{tabular}

a) $p=0.002$; the relationship between the improvement of bone continuity and that of bone alignment was tested by the Pearson chi-square test.
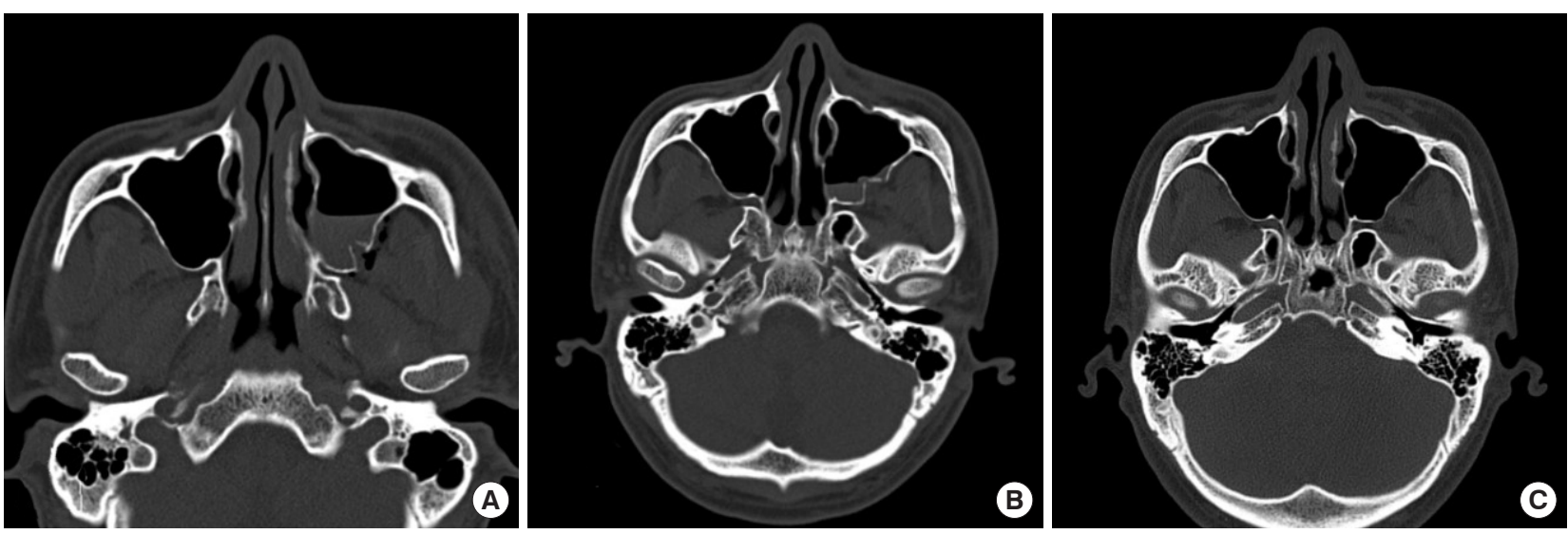

Fig. 3. Comparison of preoperative, immediate postoperative, and 3-month postoperative computed tomographic images. (A) Preoperative image showing a maxillary fracture. (B) Immediate postoperative image. (C) Three-month postoperative follow-up image. Both bone alignment and continuity had improved. 
by different genetic and epigenetic factors from those affecting other bones, resulting the presence of many cavities in the craniofacial area, including the paranasal sinuses. This mechanism of facial bone control through which injured cavities are restored may contribute to the self-healing process of facial bone fractures. Turning to other hypotheses, the amount of periosteum is much richer in the facial bone area than in other areas, which might contribute to bone healing. Claims have been made about the bone healing potential of the periosteum since the 17th century. Lin et al. [16] conducted molecular investigations into facial bone healing by the periosteum. Jung et al. [17] proposed a hypothesis according to which medial orbital wall remodeling takes place along its periosteum. The ratio of periosteum tissue to bone tissue of the facial bones is much higher than that of bones in the extremities [18]. This might contribute to self-healing of the membranous parts of the facial bones. Other factors, such as positive air pressure from sinus ventilation and muscle actions such as posterior pulling by the pterygoid muscles, may help the self-healing process. Paranasal sinus pressure rises during expiration [19]. The lower head of the lateral pterygoid muscle and the deep head of the medial pterygoid muscle originate from the lateral pterygoid plate and may be able to affect the location of the fragmented posterior maxillary wall. These actions might have contributed to the increase in maxillary volume observed 3 months after surgery.

Maxillofacial reconstruction surgery focuses on rebuilding the original shape of the affected region [20]. All functions of the upper jaw-including ventilation through the paranasal sinus system, chewing, biting, and swallowing — are associated with the original shape of the maxillary bone [20,21]. Maxillary and zygomaticomaxillary complex fracture reduction focuses on restoring the malar prominence, zygomatic arch, and maxillary buttresses [22]. The results of the present study imply that even unreduced parts can heal spontaneously if the fractured part is membranous. Therefore, surgeons should be aware that the self-healing process will continue after surgery. For this reason, meticulous reduction of small fragments of the fractured thin wall may not be necessary, and instead it may be better to focus on preserving the periosteum, which induces self-healing. Furthermore, dealing with these fragmented parts may increase the risk of iatrogenic injury of the original anatomical features. It is easy to injure the maxillary septa and arteries distributed around and inside the maxillary sinus because of limited visibility. Iatrogenic injuries of these structures may increase the complication rate $[23,24]$.Therefore, concentrating on reducing the buttress and preserving the periosteum would be much more important for promoting self-healing at thin wall fracture sites.

This study has some limitations. Volume does not inherently provide any information on the shape of the maxillary sinus. Even though the data confirmed volumetric expansion of the fractured site after reduction, this does not technically mean that the shape improved. The evaluation of bone continuity and alignment play supplementary roles in this regard; nonetheless, the data on these parameters relied on a subjective analysis (albeit by facial bone experts). Other limitations include the issue that because of the retrospective nature of this study, functional aspects of the maxillary sinus, such as voice pitch, brain temperature (which might be affected by maxillary sinus ventilation), and the mucociliary effect could not be quantified. Further studies incorporating both structural and functional analyses could guide surgeons who operate on midface fractures.

In conclusion, a longitudinal analysis of CT scans demonstrated maxillary wall remodeling through self-healing, with a concomitant increase in the maxillary sinus volume and simultaneous improvements in bone alignment and continuity. It is clear that fractured maxillary thin walls in cases of complex maxillary fractures go through a natural course of healing.

\section{NOTES}

\section{Conflict of interest}

No potential conflict of interest relevant to this article was reported.

\section{Ethical approval}

The study was approved by the Institutional Review Board of Hallym University Sacred Heart Hospital (IRB No. 2018-05035-001) and performed in accordance with the principles of the Declaration of Helsinki.

\section{ORCID}

Chan Min Chung https://orcid.org/0000-0002-2755-9440

Seung Wan Tak https://orcid.org/0000-0001-7744-5559

Hyoseob Lim https://orcid.org/0000-0002-6251-8281

Sang Hun Cho https://orcid.org/0000-0001-5906-9828

Jong Wook Lee https://orcid.org/0000-0002-3244-949X

\section{REFERENCES}

1. Gallup AC, Hack GD. Human paranasal sinuses and selective brain cooling: a ventilation system activated by yawning? Med Hypotheses 2011;77:970-3.

2. Janic T, Niedzielska I. Mucociliary clearance impairment after zygomaticomaxillary-orbital fractures. Oral Surg Oral Med Oral Pathol Oral Radiol 2013;115:e6-12.

3. Ungor C, Saridogan C, Yilmaz M, Tosun E, Senel FC, Icten O. 
An acoustical analysis of the effects of maxillary sinus augmentation on voice quality. Oral Surg Oral Med Oral Pathol Oral Radiol 2013;115:175-84.

4. Top H, Aygit C, Sarikaya A, Karaman D, Firat MF. Evaluation of maxillary sinus after treatment of midfacial fractures. J Oral Maxillofac Surg 2004;62:1229-36.

5. Yoshioka N, Tomita S, Nishikawa H, Arakawa A, Sesaki S. Medial maxillary fractures revisited. J Plast Reconstr Aesthet Surg 2014;67:506-12.

6. Beretta M, Cicciu M, Bramanti E, Maiorana C. Schneider membrane elevation in presence of sinus septa: anatomic features and surgical management. Int J Dent 2012;2012:261905.

7. Rancitelli D, Borgonovo AE, Cicciu M, Re D, Rizza F, Frigo AC, et al. Maxillary sinus septa and anatomic correlation with the Schneiderian membrane. J Craniofac Surg 2015;26:1394-8.

8. Elian N, Wallace S, Cho SC, Jalbout ZN, Froum S. Distribution of the maxillary artery as it relates to sinus floor augmentation. Int J Oral Maxillofac Implants 2005;20:784-7.

9. Solar P, Geyerhofer U, Traxler H, Windisch A, Ulm C, Watzek G. Blood supply to the maxillary sinus relevant to sinus floor elevation procedures. Clin Oral Implants Res 1999;10:34-44.

10. Traxler H, Windisch A, Geyerhofer U, Surd R, Solar P, Firbas W. Arterial blood supply of the maxillary sinus. Clin Anat 1999;12: 417-21.

11. Marx RE. Bone and bone graft healing. Oral Maxillofac Surg Clin North Am 2007;19:455-66.

12. Romano PR, Caton JG, Puzas JE. The reversal line may be a key modulator of osteoblast function: observations from an alveolar bone wound-healing model. J Periodontal Res 1997;32(1 Pt 2):143-7.

13. Guo X, Guo H, Liu J. Membrane guided tissue regeneration in the treatment of bone defect. Zhongguo Xiu Fu Chong Jian Wai Ke Za Zhi 1998;12:301-3.

14. Mardas N, Dereka X, Donos N, Dard M. Experimental model for bone regeneration in oral and cranio-maxillo-facial surgery. J Invest Surg 2014;27:32-49.

15. Esteve-Altava B, Rasskin-Gutman D. Beyond the functional matrix hypothesis: a network null model of human skull growth for the formation of bone articulations. J Anat 2014; 225:306-16.

16. Lin Z, Fateh A, Salem DM, Intini G. Periosteum: biology and applications in craniofacial bone regeneration. J Dent Res 2014; 93:109-16

17. Jung S, Lee JW, Kim CH, Hwang E, Lim H, Jung SW, et al. Postoperative changes in isolated medial orbital wall fractures based on computed tomography. J Craniofac Surg 2017;28:203841.

18. Mouraret S, Von Kaeppler E, Bardet C, Hunter DJ, Chaussain C, Bouchard $\mathrm{P}$, et al. The potential for vertical bone regeneration via maxillary periosteal elevation. J Clin Periodontol 2014;41: 1170-7.

19. Zhu JH, Lim KM, Thong KT, Wang de Y, Lee HP. Assessment of airflow ventilation in human nasal cavity and maxillary sinus before and after targeted sinonasal surgery: a numerical case study. Respir Physiol Neurobiol 2014;194:29-36.

20. Jiang FF, Hou Y, Lu L, Ding XX, Li W, Yan AH. Functional evaluation of a CAD/CAM prosthesis for immediate defect repair after total maxillectomy: a case series of 18 patients with maxillary sinus cancer. J Esthet Restor Dent 2015;27 Suppl 1:S80-9.

21. Jinfeng L, Jinsheng D, Xiaohui W, Yanjun W, Ningyu W. The pneumatization and adjacent structure of the posterior superior maxillary sinus and its effect on nasal cavity morphology. Med Sci Monit 2017;23:4166-74.

22. Tent PA, Juncar RI, Lung T, Juncar M. Midfacial fractures: a retrospective etiological study over a 10-year period in Western Romanian population. Niger J Clin Pract 2018;21:1570-5.

23. Maridati P, Stoffella E, Speroni S, Cicciu M, Maiorana C. Alveolar antral artery isolation during sinus lift procedure with the double window technique. Open Dent J 2014;8:95-103.

24. Stacchi C, Lombardi T, Cusimano P, Berton F, Lauritano F, Cervino $\mathrm{G}$, et al. Bone scrapers versus piezoelectric surgery in the lateral antrostomy for sinus floor elevation. J Craniofac Surg 2017;28:1191-6. 